\title{
ACTIVATION DETECTION IN EVENT-RELATED FMRI THROUGH CLUSTERING OF WAVELET DISTRIBUTIONS
}

\author{
Geert Verdoolaege and Yves Rosseel \\ Department of Data Analysis, Ghent University, \\ Henri Dunantlaan 1, 9000 Gent, Belgium
}

\begin{abstract}
We propose a new method for the detection of activated voxels in event-related BOLD fMRI data. We model the statistics of the wavelet histograms derived from each voxel time series independently through a generalized Gaussian distribution (GGD). We perform $k$-means clustering of the GGDs characterizing the voxel data in a synthetic data set, using the symmetrized Kullback-Leibler divergence (KLD) as a similarity measure. We compare our technique with GLM modeling and with another clustering method for activation detection that directly uses the wavelet coefficients as features. Our method is shown to be considerably more stable against realistic hemodynamic variability.
\end{abstract}

Index Terms-fMRI, $k$-means clustering, generalized Gaussian distribution, Kullback-Leibler divergence

\section{INTRODUCTION}

In neuroscience, functional magnetic resonance imaging (fMRI) has become one of the most widely used techniques for the mapping of brain activity that is related to some cognitive task. However, various sources of noise are present in the measured BOLD signal and the signal-to-noise ratio (SNR) in activated areas is typically low. This renders the detection of activated voxels a challenging task and many data analysis techniques, both hypothesis- and data-driven, have been proposed in the past to accomplish this.

The BOLD response is often modeled through the convolution of a neural activation signal with a hemodynamic response function (HRF). The general linear model (GLM) is a well-known tool in statistics that is frequently used for fMRI activation detection [1]. The GLM basically compares the acquired BOLD time series to a predefined set of basis functions. In many cases it can be useful to incorporate some $a$ priori knowledge about the expected hemodynamic response into the basis functions, hence constructing a so-called informed basis. However, it has been shown that there can be a considerable variability in the HRF from one brain region to another, or even in the same region on a trial-by-trial basis [2]. In such a case it may be better to use a more generic set of basis functions and in this respect the wavelet basis is particularly well suited. Indeed, it has been demonstrated that wavelets provide a natural framework for the analysis of neurobiological signals, which often exhibit fractal properties and nonstationary behavior of interest at multiple scales (see [3], which discusses other application of wavelets in fMRI data analysis as well). This provides our motivation for characterizing fMRI data through features in the wavelet domain.

Although many fMRI data analysis schemes, such as the GLM, operate on a voxelwise basis, there are two main disadvantages attached to this mode of operation. First, conducting statistical tests in a mass-univariate way poses a nontrivial multiple comparisons problem. Second, a voxelwise method does not take into account any spatial correlation structure within the brain and is unable to detect activation patterns. In this paper, we use a data-driven clustering approach of event-related fMRI time series, which does not suffer from the above drawbacks. Cluster analysis of fMRI data has been considered before, but the employed features are often directly linked to the raw time series or they depend on a specific HRF model (see [4] and references therein). Hence, such features are in general less robust against HRF variability, as we will show in the present work. For instance, in [5] clustering was performed on a subset of the detail coefficients resulting from a discrete wavelet transform of the voxel time series (which we will refer to as the method 'COEF'). The clustering method that we propose, which we here call the method 'STAT', is conceptually simple and is also based on wavelet features. However, instead of clustering directly on the wavelet coefficients, we use the statistics of wavelet detail coefficients as an indicator for activation. We model the wavelet statistics via a generalized Gaussian distribution (GGD) and we evaluate GGD similarity through the symmetrized Kullback-Leibler divergence (KLD). The KLD is subsequently applied in a $k$-means clustering algorithm. In a comparison of our technique with both COEF and classic GLM modeling, we show that the use of wavelet statistics leads to an enhanced stability of the method against hemodynamic variability. We work with an artificial data set, which has the advantage that the activation pattern is known a priori, facilitating comparison of different activation detection strategies.

This contribution is organized as follows. In Section 2 we 
present the design of our experiments: the generation of the artificial data set, the statistics of the wavelet coefficients and GGD similarity measurement via the KLD in a $k$-means clustering scheme. In Section 3 we discuss the results of the clustering experiments for activation detection using STAT and we compare with the GLM and COEF. Section 4 provides a conclusion and an outlook towards future work.

\section{DESIGN OF CLUSTERING EXPERIMENTS}

\subsection{Synthetic data set}

We demonstrate activation detection on an artificial data set consisting of a volume of $14 \times 14 \times 14$ voxels, every voxel containing a time series of 336 samples. A repetition time (TR) of $2 \mathrm{~s}$ was chosen. Activation was introduced in half of the number of time series (voxels), consisting of 24 regularly timed block stimuli of $4 \mathrm{~s}$ duration each. The BOLD response corresponding to this activation was obtained by convolving with the HRF. In a first experiment a fixed canonical HRF throughout the simulated volume was assumed. The canonical HRF $h(t)$ provides a physiologically reasonable model for a typical hemodynamic response [2]. It is defined as follows:

$h(t)=\left(\frac{t}{d_{1}}\right)^{a_{1}} \exp \left(\frac{d_{1}-t}{b_{1}}\right)-c\left(\frac{t}{d_{2}}\right)^{a_{2}} \exp \left(\frac{d_{2}-t}{b_{2}}\right)$.

Here, $d_{1}=a_{1} b_{1}$ is the time associated to the maximum of the initial peak and $d_{2}=a_{2} b_{2}$ is the time to the minimum of the undershoot. We started from typical values for the parameters: $a_{1}=6, a_{2}=12, b \equiv b_{1}=b_{2}=0.9$ and $c=0.35$. The corresponding function $h(t)$ is drawn in Figure 1. A second experiment was designed in order to study the robustness of the activation detection scheme against spatial hemodynamic variability. To this end a fluctuation was introduced in the HRF dispersion (parameter $b$ ) and amplitude of the undershoot $(c)$. In addition, a variable response latency was imposed by delaying the HRF by a time lag $D$. The values of $b, c$ and $D$ were chosen at random from voxel to voxel, by sampling from the following truncated Gaussian distributions:

$\begin{array}{rrrr}b: & \mathcal{N}(0.9,1.0) & \wedge & 0.5 \leq b \leq 2.0, \\ c: & \mathcal{N}(0.35,1.0) & \wedge & 0.0 \leq c \leq 1.0, \\ D: & \mathcal{N}(0.0,1.5) & \wedge & 0.0 \leq D \leq 2.0\end{array}$

where $\mathcal{N}(\mu, \sigma)$ signifies the normal distribution with mean $\mu$ and standard deviation $\sigma$. This represents a realistic hemodynamic variability supported by empirical data [2]. Apart from the canonical HRF, some more example HRFs are drawn in Figure 1. No trial-to-trial HRF variability was assumed.

Several models of varying complexity for the noise in fMRI data are commonly used. In the present experiments

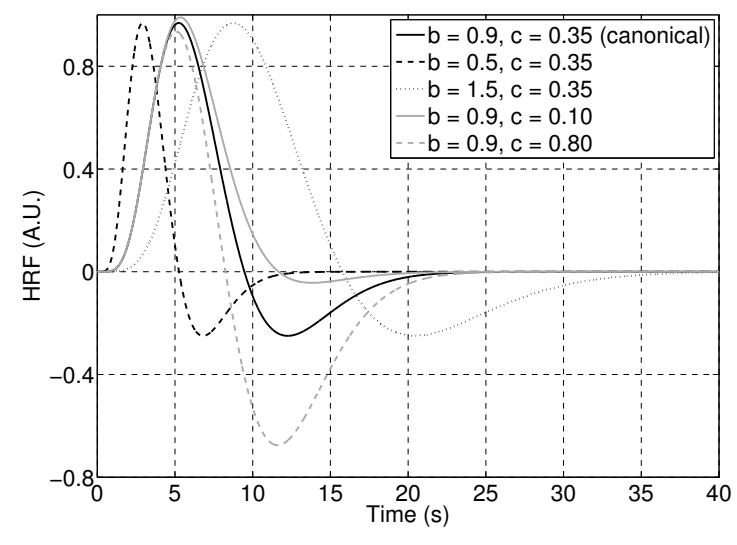

Fig. 1. Canonical HRF and examples of other HRFs for some extreme values of the parameters in the HRF model that were varied from voxel to voxel.

we simply assumed additive Gaussian noise leading to a relatively low SNR. The (functional) SNR in fMRI is usually mentioned as the ratio of the standard deviation $\sigma_{\mathrm{S}}$ of the pure signal (activation), to the standard deviation $\sigma_{\mathrm{n}}$ of the noise. Typical values are $\sigma_{\mathrm{s}} / \sigma_{\mathrm{n}} \lesssim 1$, while we assumed an SNR of 0.6 (approximately $-4 \mathrm{~dB}$ ). The non-activated time series contained only noise with the same standard deviation. An example of a simulated time series, showing both the pure and the noisy signal, is given in Figure 2.

\subsection{Wavelet statistics}

Applied to our synthetic data set, the purpose of an activation detection scheme based on clustering is to discriminate between the activated half of the simulated volume and the non-activated part. The selection of features used for our clustering method STAT was conducted as follows. First, the redundant discrete wavelet transform (RDWT) was applied independently to each time series, using Daubechies' 4-tap wavelets. An example of the RDWT of an artificial activated time series (BOLD response + noise) is presented in Figure 2, showing the first four levels. The effect of the original BOLD response can easily be discerned at the higher wavelet detail scales. The RDWT results in a number of wavelet detail coefficients sufficient to adequately characterize the wavelet statistics. Wavelet detail histograms, which have zero mean, often exhibit a distinctly non-Gaussian shape. For instance, in [6] wavelet coefficients for gray-level images were modeled in an image retrieval context using a univariate zeromean generalized Gaussian distribution, also known by the name of exponential power distribution. The GGD in general provided a better fit than the Gaussian density. The univariate zero-mean GGD is defined through the following density function:

$$
p(x \mid \alpha, \beta)=\frac{\beta}{2 \alpha \Gamma(1 / \beta)} \exp \left[-\left(\frac{|x|}{\alpha}\right)^{\beta}\right],
$$




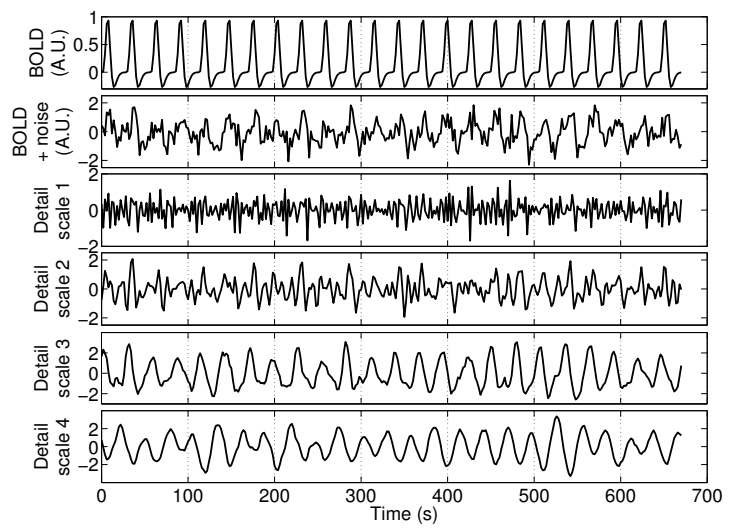

Fig. 2. The BOLD response (top frame) as a result of a synthetic regular series of block stimuli (4 s each), employing the canonical HRF. The second frame shows the same signal with added Gaussian noise $(-4 \mathrm{~dB})$. In the following frames the wavelet detail coefficients are plotted corresponding to the first four levels of the RDWT of the noisy signal using the Daubechies 4-tap wavelets.

where $\Gamma($.$) represents the Gamma function. \alpha$ models the width of the density function (related to the standard deviation), while $\beta$ is connected to its kurtosis. Clearly, the Gaussian distribution is obtained by setting $\beta=2$.

Example histograms of the redundant wavelet detail coefficients at the fourth scale obtained from both a non-activated and an activated times series (same data as in Figure 2), are displayed in Figure 3. The respective GGD fits as well as the best-fit Gaussian distributions are also shown and it can be seen that the GGD captures the wavelet statistics much better than the Gaussian. Accordingly, we described the wavelet detail coefficients corresponding to the time series in our artificial volume at the first four levels independently through a set of univariate GGDs. The choice of wavelet scales largely depends on the nature of the signal, but in our application we found that including the first scale as well as larger scales than the fourth had little impact on clustering performance, so we ignored them in our detection scheme. This measure is, in addition, beneficial for the COEF method since it substantially limits the number of features. The features that we used to characterize each voxel time series are thus given by the parameters $\alpha$ and $\beta$ of the univariate GGDs modeling the wavelet statistics. Since we routinely employed three wavelet scales (scales two to four) we obtained six features per voxel, representing a dramatic reduction of dimensionality.

\subsection{Clustering}

The next step in our STAT method involved the clustering of voxels on the basis of their corresponding GGD features. An overview and comparison of several clustering algorithms in the context of fMRI cluster analysis was given in [4]. The su-

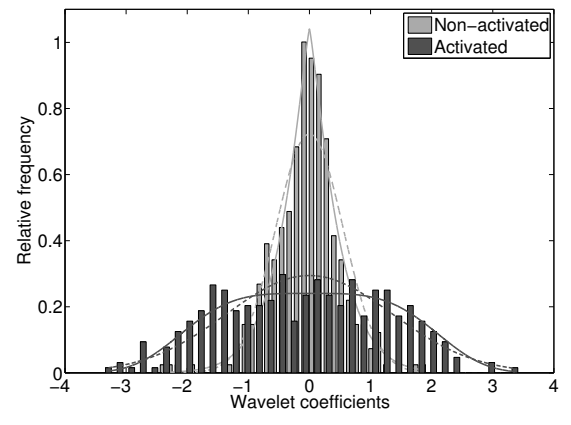

Fig. 3. Histograms for the redundant wavelet detail coefficients at level four corresponding to a time series containing only noise (non-activated) and a time series in an activated voxel. The GGD fits are displayed as well (full lines) together with the best-fit Gaussian density functions (dashed lines).

perior clustering performance of the $k$-means and neural gas algorithms was demonstrated, compared to other well-known techniques. For this reason in the present study we applied the $k$-means algorithm, which in addition is simple to implement. The $k$-means algorithm assumes the number of clusters $k$ is known and represents the clusters by their centroid. The aim is to minimize the within-cluster sum-of-squares. In our case $k=2$ since we simply distinguish between activated and non-activated voxels. The initialization phase of the algorithm consists of assigning random cluster centroids. The algorithm next proceeds iteratively by cycling through two steps: first, assign each point to the cluster with the nearest centroid. Then, for every obtained cluster recalculate the centroid. The clustering efficiency depends partly on the choice of similarity measure, for which we took the symmetrized Kullback-Leibler divergence. A closed-form expression exists for the KLD between univariate zero-mean GGDs [6], rendering our implementation of the $k$-means algorithm sufficiently fast. In addition, the wavelet scales were assumed to be independent, so that the total KLD between voxels could simply be obtained by summing the KLDs between corresponding scales [6]. The cluster centroids were defined by the Karcher mean of the cluster points, again using the symmetrized KLD as a similarity measure.

\section{RESULTS AND DISCUSSION}

As mentioned in the introduction, we compared our technique to the method COEF, for which we used as features the wavelet detail coefficients in scales two to four obtained with the DWT (including subsampling). In COEF the Euclidean distance between wavelet coefficients is applied in a $k$-means clustering algorithm. In both the case of COEF and STAT, we performed the $k$-means cluster analysis several times in order to study the effect of random initialization of cluster centroids, which turned out to be negligible. A typical set of clusters in GGD parameter space is shown in Figure 4, together 


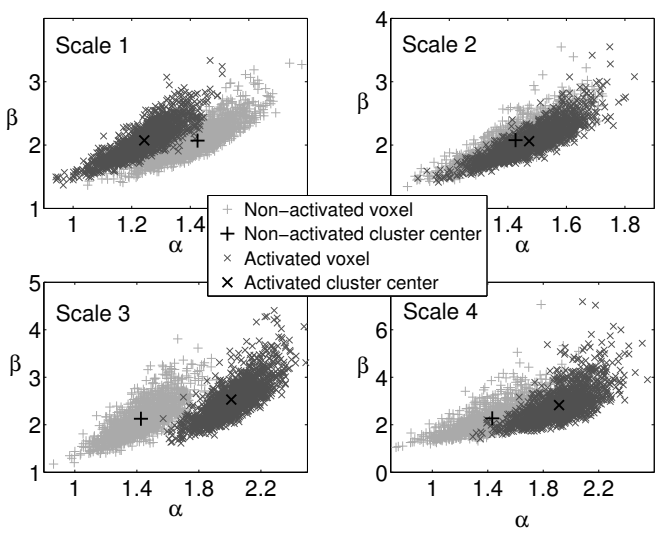

Fig. 4. GGD parameters, displayed independently for the first four scales of the RDWT, corresponding to the voxel time series in the synthetic volume (each point represents a voxel). Cluster centroids obtained via a $k$-means clustering algorithm are also indicated.

with the cluster centroids found by the $k$-means algorithm. A clear non-Gaussian behavior can be observed of the wavelet statistics in many voxels. It should be noted that the cluster shapes do not necessarily reflect the true similarity structure of the voxels, since the plot is in Euclidean space while the applied similarity measure is non-Euclidean (KLD).

The results from the two experiments (fixed and variable HRF) are summarized in Table 1 in terms of false positive rates (FPR) and true positive rates (TPR). For comparison with the performance of the GLM, using the canonical HRF as a model, the TPR for a given FPR was determined from the distributions of the test statistic for the non-activated and activated voxels. Since for both STAT and COEF the FPR turns out to lie below $1 \%$, we chose to mention the TPR obtained by the GLM corresponding to an FPR of $0.1 \%$ and $1 \%$.

All three methods perform very similarly in case of a fixed canonical HRF throughout the simulated volume. However, when HRF variability is introduced, the FPRs remain stable but our STAT method exhibits a substantially higher TPR than both the GLM and the COEF method. Hence, STAT turns out to be much more robust against HRF variability compared to the GLM and COEF.

\section{CONCLUSION}

We have proposed a new data-driven method for activation detection in event-related BOLD fMRI. The technique is based on $k$-means clustering of the parameters of a generalized Gaussian distribution, fit to wavelet detail coefficients obtained from the fMRI time series via the redundant discrete wavelet transform. We have compared our method to classic GLM modeling and to a clustering-based scheme that uses raw wavelet coefficients as features. Our method turns out to

\begin{tabular}{lcccc}
\hline & & GLM & COEF & STAT \\
\cline { 2 - 5 } Canonical HRF & FPR $(\%)$ & $0.1 / 1.0$ & 0.1 & 0.3 \\
& TPR $(\%)$ & 100 & 100 & 100 \\
\hline \multirow{2}{*}{ Variable HRF } & FPR $(\%)$ & $0.1 / 1.0$ & 0.0 & 0.0 \\
& TPR $(\%)$ & $83.3 / 88.1$ & 54.7 & 92.5 \\
\hline
\end{tabular}

Table 1. False positive rates (FPR) and true positive rates (TPR) in the case of a fixed and variable HRF in an experiment on an artificial fMRI volume using the methods GLM, COEF and STAT.

be considerably more stable against hemodynamic variability, which is ubiquitous in real fMRI data. A similar robustness against uncertainty in stimulus timing is to be expected (useful for e.g. resting-state experiments). This will be the subject of further study, as well as the use of other clustering algorithms (e.g. Gaussian mixture modeling) and GGD similarity measures (e.g. the geodesic distance).

\section{REFERENCES}

[1] K.J. Friston, A.P. Holmes, K.J. Worsley, J.-B. Poline, C.D. Frith, and R.S.J. Frackowiak, "Statistical Parametric Maps in functional imaging: A general linear approach," Hum. Brain Mapp., vol. 2, pp. 189-210, 1994.

[2] K.J. Friston, P. Fletcher, O. Josephs, A. Holmes, M.D. Rugg, and R. Turner, "Event-related fMRI: Characterizing differential responses," Neuroimage, vol. 7, pp. 3040, 1998 .

[3] E. Bullmore, J. Fadili, V. Maxim, L. Xendur, B. Whitcher, J. Suckling, M. Brammer, and M. Breakspear, "Wavelets and functional magnetic resonance imaging of the human brain," Neuroimage, vol. 23, pp. S234-S249, 2004.

[4] E. Dimitriadou, M. Barth, C. Windischberger, K. Hornik, and E. Moser, "A quantitative comparison of functional MRI cluster analysis," Artif. Intell. Med., vol. 31, pp. 5771, 2004.

[5] B. Whitcher, A.J. Schwarz, H. Barjat, S.C. Smart, R.I. Grundy, and M.F. James, "Wavelet-based cluster analysis: data-driven grouping of voxel time courses with application to perfusion-weighted and pharmacological MRI of the rat brain," Neuroimage, vol. 24, pp. 281-295, 2005.

[6] M.N. Do and M. Vetterli, "Wavelet-based texture retrieval using generalized Gaussian density and KullbackLeibler distance," IEEE Trans. Image Process., vol. 11, pp. 146-158, 2002. 